\title{
Il femminile matriarcale nella narrativa di Marisa Madieri
}

\author{
Daniele Cerrato $^{1}$
}

Recibido: 19 de enero de 2016 / Aceptado: 17 de marzo de 2016

Riassunto. Questo articolo esamina ed interpreta la narrativa della scrittrice italiana Marisa Madieri come un riscatto e una riscoperta del femminile matriarcale. La presenza di una genealogia femminile rappresenta un elemento costante all'interno dei suoi testi, dove sono presenti numerosi archetipi legati direttamente al mito della Grande Madre o della Dea Madre. Sono, inoltre, molto frequenti le metafore ricollegabili al femminile come forza generatrice e i riferimenti a figure mitologiche e simboli del femminile.

Parole chiave: Marisa Madieri; genealogia; matriarcato; Grande Madre; mitologia.

\section{[en] The feminine matriarchal in the Marisa Madieri's narrative}

\begin{abstract}
This paper examines and interprets the narrative of the Italian woman writer Marisa Madieri as a rediscovering of the matriarchal female. The building of a genealogy represents a constant element within her writings. Many of these are the female archetypes directed linked to the Great Mother or Great Godness myth. In addition, there are many female metaphors that are related to the generative force and references to female mythological figures and female symbols.
\end{abstract}

Key words: Marisa Madieri; genealogy; matriarchy; Great Mother; mitology.

Sommario: 1. Introduzione 2. La ricostruzione di una genealogia femminile 2.1 Genealogia femminile come sororità 3. La lingua madre 4. La rappresentazione della natura: il giardino e la terra-madre 5. La rilettura del mito: Dafne, la sirena e altri archetipi della Dea Madre 6. Acqua, mare, grotte e conchiglie.

Come citare: Cerrato, Daniele (2016): «Il femminile matriarcale nella narrativa di Marisa Madieri», Cuadernos de Filología Italiana, 23, pp. 155-173.

\section{Introduzione}

Durante un congresso celebratosi nel 2001 a Moguer, Ernestina Pellegrini (2001: 605-618) ricordava come l'opera di Marisa Madieri potrebbe essere contenuta facilmente in una grande tasca. Eppure, quelle circa trecento pagine tra il diario-

1 Universidad de Sevilla. Facultad de Filología, Departamento de Filologías Integradas, Calle Palos de la Frontera s/n, 41004, Sevilla, España.

dcerrato@us.es 
memoria Verde Acqua, il racconto fiaba La Radura, il romanzo incompiuto Maria, e i racconti de La Conchiglia, non solo sono state sufficienti a conquistare negli anni molti lettori, ma hanno anche stimolato un dibattito all'interno della critica letteraria, producendo una vasta bibliografia sull'autrice e sulla sua opera ${ }^{2}$.

Verde Acqua, pubblicato nel 1987 con Einaudi, ottenne un'ottima accoglienza, e Geno Pampaloni (1987) lo considerò «un piccolo classico contemporaneo della memoria familiare», mentre, successivamente, Enzo Siciliano (2001) lo ha definito «un tessuto di ombre e luci, di quel morire e rinascere che è la vita». Il testo ha raccolto molti consensi anche all'estero ed è stato tradotto in moltissime lingue (croato, spagnolo, francese, tedesco, polacco, sloveno, ceco, lituano, catalano, slovacco) e celebrato come «una de esas joyas inclasificables que contiene cada literatura» (Monmany 2001).

Lo stesso dicasi per gli altri scritti dell'autrice che sono stati studiati in diversi paesi $^{3}$, da La Radura, «una storia semplice come la vita in cui tornano tutte le elementari verità dell'esistere» (Paccagnini 1998: 17), al racconto La Conchiglia, «un piccolo capolavoro a cui non si potrebbe aggiungere né togliere una virgola» (Magris 1998: 288), fino a Maria, ultimo testo pubblicato, storia che «turba nella sua forza devastante» (Torossi Tevini 2007: 14).

Nel corso degli ultimi decenni, si sono analizzati diversi aspetti della produzione di Marisa Madieri, sia per quanto riguarda lo stile, sia per quanto concerne i contenuti. Si è detto, ad esempio, che l'autrice ha saputo dare vita ad un'opera «originale, straordinariamente ricca di rimandi, di sguardi, di voci, di storie plurali, che intersecano le inquietudini della cultura contemporanea» (Carminati 2006: 176) e di come tutta la sua produzione si può leggere come «una lunga meditazione sulla vita e sulla morte, una meditazione e contemplazione che hanno qualcosa di meraviglioso ed incantatorio, di fiero ed insieme di malinconico» (Pellegrini 2001: 173). Si è fatto, inoltre, riferimento alla «limpidezza e semplicità» della sua scrittura (Gerbaz 2011: 65) e di come «il senso del suo lavoro [...] è stato quello del cavare, del togliere, del ridurre all'osso il discorso liberandolo da tutti quegli ornamenti che spesso vengono indebitamente scambiati - da critici e scrittori - per una caratteristica del lavoro letterario» (Guagnini 2004).

Restano, in ogni caso, alcuni aspetti della produzione della scrittrice che fino ad ora si sono analizzati solo in parte e che possono essere approfonditi e costituire il punto di partenza per nuovi studi e proposte di ricerca.

2 Tra le studiose e gli studiosi che si sono occupati di Maria Madieri si possono ricordare, oltre ad Ernestina Pellegrini e ovviamente Claudio Magris (1998), Geno Pampaloni (1987), Gabriele Benelli (1993), Elvio Guagnini (1994), Ermanno Paccagnini (1998), Mercedes Monmany (2001), Enzo Siciliano (2001), Graziano Bianchi (2003), Maria Carminati (2007), Fabio Russo (2007), Cristina Benussi e Graziella Semacchi Gliubich (2011), Corinna Gerbaz Giuliano e Gianna Mazzieri Sanković (2013). A Trieste si è organizzata (10 dicembre 2014 - 24 gennaio 2015) la mostra documentaria «Piccole gocce nell'oceano del vissuto: Marisa Madieri e l'emozione della scrittura», a cura di Elvio Guagnini, Pedro Luis Ladrón de Guevara e Anna Storti, mentre all'Università di Murcia, ad aprile 2015, si è svolto il Congresso Internazionale «Omaggio a Marisa Madieri: scrittrici dell'esodo e dell'esilio» organizzato da Pedro Luis Ladrón De Guevara.

3 La Radura è stato tradotto in croato, spagnolo e francese, il racconto La conchiglia in quattordici lingue (albanese, bulgaro, ceco, croato, finlandese, greco moderno, inglese, polacco, russo, slavo-macedone, sloveno, slovacco, ucraino, ungherese). Per una bibliografia aggiornata sulle traduzioni dei testi di Marisa Madieri e sugli studi relativi alla sua opera si rimanda a Zografidou (2015: 195-203). 
Sulla linea di quanto già affermava Ernestina Pellegrini (1995), che sottolineava la scelta di Marisa Madieri di valorizzare la saggezza e la pazienza femminili, e di farne un patrimonio culturale e della tradizione, l'intera opera dell'autrice può essere interpretata come un riscatto e una riscoperta del 'femminile', oltre a rappresentare, attraverso simboli e metafore, un universo che si rifà al matriarcato ${ }^{4}$.

L'obiettivo di questo articolo è perciò quello di studiare il femminile matriarcale all'interno dell'opera di Marisa Madieri, analizzando come questo concetto si sviluppa attraverso differenti personaggi, miti ed immagini.

Con femminile matriarcale si vuole fare riferimento sia al ruolo che alle pratiche delle donne all'interno della società e della famiglia e, quindi, alla capacità di costruire e rinforzare legami di genealogia e "sororità", sia alla presenza di archetipi del femminile legati direttamente al mito della Grande Madre o della Dea Madre.

I testi di Marisa Madieri si possono prestare a questo tipo di analisi, perché restituiscono alle donne quella soggettività di cui sono state private nel corso dei secoli, e rivendicano il fondamentale contributo femminile alla costruzione della piccola e grande storia. Come osserva Claudio Magris, la narrativa di Marisa Madieri racchiude, infatti, «l'esilio femminile, l'atopia, il non luogo della donna nella Storia $[\ldots]$ ma anche una riappropriazione non ostentata della femminilità, un viaggio verso la Storia attraverso l'astoricità dell'oppressa condizione femminile» (Magris 1998: 293).

Gesti e aspetti del quotidiano assumono una nuova forma e prospettiva, che li eleva a mito e a simbolo. Il ruolo delle donne all'interno della società, tradizionalmente giudicato come marginale e/o naturale, e quindi, invisibile e silenziato, diventa nella narrativa di Marisa Madieri uno degli aspetti di maggiore rilievo, come la stessa autrice non ha mancato di sottolineare:

Mi interessa la vita minore, ciò che resta appunto al margine della storia e dell'ideologia, la vita che non può parlare, far sentire la propria voce; questo profondo interesse per tutto ciò che è minimo, è ai margini, alla periferia della vita, in qualche modo escluso dalla Storia... è una componente essenziale della mia visione del mondo. (Madieri 2007: 75)

\section{La ricostruzione di una genealogia femminile}

Nei documenti ed appunti inediti di Marisa Madieri, pubblicati recentemente ${ }^{5}$, l'autrice osservava come le donne

per il loro ruolo storico sono ancora oggi depositarie della pietas virgiliana, hanno più attenzione per il dettaglio, per il piccolo, per il debole, per la fugace $\mathrm{e}$ umile quotidianità rispetto ad una cultura «maschile» del progetto, della

4 Sul matriarcato, sulla simbologia legata alla Grande Madre e sul significato delle sue rappresentazioni, si vedano ad esempio, Bachofen (1861), Neumann (1956), Gimbutas (1989).

5 I documenti inediti di Marisa Madieri sono riuniti in un'appendice, corredati dalla loro traduzione in spagnolo, in Ladrón de Guevara et al. (2015: 163-185). 
conquista del futuro che deve fatalmente sacrificare l'individuo alla società, la parte al tutto, l'oggi al domani. (Madieri 2015: 170)

Nella sua narrativa, oltre ad essere portatrici della memoria e della storia, sono protagoniste del riscatto del femminile attraverso il recupero di una linea matriarcale e la ricostruzione di una genealogia di donne.

Secondo Rossana Rossanda ${ }^{6}$, Verde Acqua ne è un chiaro esempio, e anche Ernestina Pellegrini considera quest'opera una straordinaria storia di matriarcato «pietoso ma a volte feroce» (Pellegrini 1995: 133). Tutto il diario-memoria ruota intorno a figure femminili e sono soprattutto le donne più anziane della famiglia a ricoprire un ruolo di primo piano. Non è certo un caso che il romanzo si apra con la descrizione della nonna paterna, 'nonna Madieri':

Era alta, diritta e silenziosa. I suoi occhi, con le palpebre rigonfie, erano due fessure leggermente piegate all'ingiù, la bocca sottile e dura. Il volto, dai lineamenti imperiosi, era addolcito da una nuvola di capelli soffici e bianchi, con qualche striatura giallastra, raccolti a chignon sulla nuca. Quando mi teneva in braccio tuffavo il volto tra quei capelli, il cui odore di pulito è legato per me ancora oggi al suo ricordo. (Madieri 1998: 21)

Oltre a Nonna Madieri, nel testo compaiono la Nonna materna (Nonna Quarantotto) e Nonna Anka, ultima compagna del padre di Marisa, divenuta una nonna acquisita e parte integrante della famiglia.

Anka Grkovic, vedova Puhalj, vedova Belić, vedova Gregorutti e di fatto anche vedova Madieri, è stata cooptata nella nostra famiglia e promossa nonna dopo essere diventata a settant'anni passati l'ultima compagna di mio padre, vedovo anche lui da un paio d'anni e diventato ormai per la famiglia nonno Gigio. [...] Nonna Anka vuole di riflesso molto bene a me a mia sorella e alle nostre famiglie, considera i nostri figli i suoi nipoti ed ha soprattutto un debole per Paolo perché tanto somigliante a Nonno Gigio. (Madieri 1998: 39)

Nonna Anka è una figura che svolge un ruolo importante nel recupero e conservazione della genealogia femminile della famiglia. Attribuisce grande importanza al passato e al ricordo e culto dei defunti, come dimostra la maniera di prodigarsi per mantenere viva la memoria di mamma Jole: «sul pianoforte c'è una fotografia della mamma, davanti alla quale nonna Anka ha posto un mazzetto di fiori finti. Non l'ha mai conosciuta ma ne custodisce la memoria con venerazione, poiché non rinnega nulla del passato» (Madieri 1998: 153).

Sarà sempre Nonna Anka a tracciare una sorta di linea di sororità tra nonne, collocando un quadro di Nonna Madieri nella casa di via Piccardi.

Su una parete del salotto di via Piccardi, $[\ldots]$ è appeso un ritratto a olio, in grandezza naturale, della nonna Madieri, bella e imperiosa, i capelli bruni

6 L'intervento di Rossana Rossanda avvenne durante un incontro svoltosi a Roma il 21 novembre 1998 presso l'Associazione Civita. Rossana Rossanda sottolineava, inoltre, come l'esilio descritto in Verde Acqua è in un certo senso, anche la rappresentazione dell'esilio della donna dalla storia, aggiungendo come «c'è chi vive storicamente e chi no queste vicende, in entrambi i casi si guadagna e si perde qualcosa». 
raccolti gonfi sul capo, alta e slanciata la figura. È stata nonna Anka a ripescare in cantina questa tela ingombrante e farla incorniciare. Così gli enigmi di quel volto, del suo passato e delle mie origini mi vengono riproposti ogniqualvolta entro in quella stanza e incontro quello sguardo che segue dovunque l'osservatore. Non riesco quasi più ad immaginare anziana questa nonna, raffigurata nella sua splendida maturità. Se non fosse per i candidi capelli profumati di pulito che ricordo chiaramente, mi sembrerebbe di averla conosciuta solo così, ancor giovane, con la vita sottile, la camicetta orlata di pizzo, un filo di perle attorno al collo ed una mano nascosta dietro la gonna lunga. (Madieri 1998: 170)

Tracce di un femminile matriarcale si possono riscontrare anche quando, all'interno del Silos, si decide di allestire una cappella e Nonna Quarantotto ne diventa la responsabile, svolgendo con autorità e orgoglio l'attività di sacerdotessa. L'autrice lo racconta, non senza un velo d'ironia.

La nonna, che in vita sua era stata tutt'altro che devota s'improvvisò sagrestano. Divenne custode degli arredi sacri e responsabile del decoro della cappella. Rinnovava i fiori, accendeva le candele e preparava l'altare prima delle funzioni. Imparò anche a biascicare in latino le risposte da dare alle invocazioni dell'officiante durante la messa. In cuor suo probabilmente riteneva di essere lei il vero ministro del culto. Considerò un trionfo personale l'arrivo al Silos della Madonna Pellegrina. (Madieri 1998: 138)

In Verde Acqua, Nonna Quarantotto incarna in parte un femminile negativo, ma anche se la narrazione sottolinea la sua ansia di protagonismo e il suo despotismo, non si tratta di un ritratto completamente a tinte fosche, dal momento che la sua intelligenza, la sua vitalità e intraprendenza ne fanno un personaggio sui generis, epico ed affascinante, che finisce per suscitare un fondo di simpatia ed ammirazione.

La figura femminile più importante e decisiva di Verde Acqua è la mamma di Marisa, mamma Jole, come l'autrice ricorda nella pagina che porta come data 6 dicembre 1981: «A mia madre penso sempre più spesso e intensamente. Le radici della mia forza e della mia capacità di non arrendermi di fronte alle difficoltà affondano nel suo amore» (Madieri 1998: 28).

Si tratta di un riconoscimento ontologico verso la figura materna, origine della vita, ma anche dell'energia vitale. D'altronde, il legame madre-figlia, come sottolinea Luce Irigaray (1989), si può considerare il rapporto più privilegiato ed evoluto, dal momento che solo la figlia, che è potenzialmente madre, può identificarsi e confrontarsi con chi l'ha generata e portata dentro di sé.

Jole si impone, ad esempio, sul marito, affinché le figlie possano continuare a studiare, impara l'alfabeto greco per aiutare Marisa durante il ginnasio, e quando si rende conto che lei vorrebbe rinunciare ad un invito di una compagna, non avendo un abito adatto da indossare, le compra quel vestito color verde acqua, a cui si richiama anche il titolo.

La mamma mi lesse nel cuore. Portò al Monte di Pietà, come aveva fatto altre volte, il suo braccialetto di metallo bianco dopo aver ben strofinato con un panno per renderlo lucido, e la sua pelliccia, probabilmente di coniglio, tutta consunta. 
Ciò le permise di comperarmi una gonna a campana e un completo formato da un cardigan e una maglia a giro collo, in orlon color verde Nilo. Conservai quel completino per anni, con gelosia, anche se purtroppo il tessuto di fibra sintetica, con le lavature, divenne sempre più lungo e più largo, fino a sformarsi del tutto. Anche verde acqua si chiamava quel colore, che per me è ancora oggi il colore dell'amore. (Madieri 1998: 146)

Non si tratta soltanto di amore materno, ma anche di un sentimento di sororità nei confronti della figlia, di una rivendicazione e voglia di riscatto possibile, proiettandosi in lei, e affidandole i sogni incompiuti di quando era ragazza.

Jole fornirà un'ulteriore prova della sua umanità e generosità quando accudirà pazientemente sua madre, la nonna Quarantotto, sopportandone il dispotismo degli ultimi anni.

La catena di solidarietà femminile e il tema della cura sono costanti nella narrativa di Marisa Madieri e si ritroveranno in Maria o nel racconto I Barattoli, più esplicitamente, ma anche ne La Radura, dove la protagonista, Dafne, ne rappresenta, in un certo qual modo, una declinazione fiabesca, dove la cura diventa generosità e disposizione naturale verso l'altro.

L'amore di madre e di figlia, che la figura di Jole racchiude, si esplicita nella sua descrizione poco prima della morte, dove si richiama la rappresentazione della Grande Madre, come anziana. Si completano, così, le tre fasi del ciclo vitale (giovane, donna, vecchia), con le quali si sottolinea anche il rapporto di reciprocità e gratitudine che esiste fra loro:

La mamma a sessant'anni morì con l'aspetto di una centenaria. [...] In quelle rughe simili ai segni che il mare lascia sulla sabbia, in quei lineamenti antichi ed irriconoscibili, in quei capelli ostinatamente folti e vigorosi, vedevo, come nel Siddartha, i solchi della terra, l'illusione del tempo, i fiumi, gli alberi e le città della mia vita, le strade che la sua carità aveva tracciato, i petali bianchi delle mie viole d'infanzia, l'amore tenace e doloroso che i suoi baci mi avevano insegnato. (Madieri 1998: 174)

Il tentativo di proteggere e conservare questa genealogia matrilineare si avverte anche nei piccoli particolari, come ad esempio nel gesto di preservare e difendere dall'usura del tempo i simboli di questo legame, non solo il vestito verde acqua, ma anche le federe dei cuscini, o una semplice spazzola. Gli oggetti della quotidianità portano le tracce delle altre donne della famiglia, prendono vita, assumendo un valore magico, che permette alla protagonista di richiamare a sé la figura materna, di esserle nuovamente vicina.

Ho cambiato questa mattina le federe dei miei cuscini, sostituendole con altre in lino, ricamate dalla mamma per il suo corredo. Ho notato con dolore che in parecchi punti il tessuto è liso e trasparente come la pelle di certi vecchi. Non le userò più, poiché non voglio che il tempo assapori troppo presto il suo ultimo trionfo. Le salverò assieme ad una spazzola per abiti in velluto rosso, a forma di gatto, che la mamma mi regalò per un mio compleanno tanti anni fa. L'ho riposta nell'angolo di un armadio, che è piuttosto un angolo segreto del mio cuore, a volte remoto e polveroso, a volte dilatato sulla vertigine degli anni trascorsi. (Madieri 1998: 103) 


\subsection{Genealogia femminile come sororità}

Un'altra declinazione della genealogia femminile si manifesta attraverso la sororità, riferita non solo al rapporto che lega le sorelle all'interno dei vari testi di Marisa Madieri, ma in alcuni casi anche alla complicità e reciprocità che si instaura tra altri personaggi femminili.

Nelle pagine di Verde acqua, Lucina, sorella di Marisa e di quattro anni più giovane, è la sua prima compagna di giochi, poi improvvisamente avviene la dolorosa separazione, a cui segue il tanto agognato ricongiungimento presso i Silos. È Lucina che le fa da guida per i labirinti stretti di quel «paesaggio vagamente dantesco» (Madieri 1998: 88), insieme condividono piccole gioie come quella di nutrire e veder crescere un piccolo passerotto caduto dal nido e portato in salvo, studiano negli spazi angusti del loro box di Trieste e poi, una volta più grandi, partecipano alle prime escursioni sul Carso e in Val Rosandra, tenendosi per mano in «reciproco sostegno» (Madieri 1998: 149).

Anche nella favola floreale La Radura i personaggi femminili predominano, e seppur la madre di Dafne non compaia, la sua presenza si avverte costantemente per tutto l'arco del racconto.

Come tutti i suoi simili Dafne non conosceva i suoi genitori, poiché questi potevano abitare molto lontano o essere addirittura sfioriti nella stagione precedente. Ma a lei talvolta pareva di sentire una voce carezzevole e materna provenire da una zona remota, seminascosta da un masso, dietro il quale sbucavano alcuni capolini e ligule bianche. «Comportatevi bene ragazze», s'illudeva di udire in quella direzione, se il vento era favorevole, oppure: «State attente a non prendere un colpo di sole. Riparatevi». (Madieri 1998: 278)

Dafne cresce circondata dalle sorelle Camilla, Amanda, Rachele, ma è soprattutto con quest'ultima, la sorella più grande, che si crea una relazione più intima, dal momento che sembra essere l'unica a comprendere le sue inquietudini. È la sola a ritenere importante che Dafne possa continuare a sognare, e quando questa le domanda a proposito delle fate ${ }^{7}$, non ne nega l'esistenza. Rachele svolge un doppio ruolo, quello di sorella e quello di mamma. Infatti, quando la notte prima del temporale, Dafne la vede piangere e, preoccupata, le chiede il motivo, per lei è naturale chiamarla «bambina mia» (Madieri 1998: 189).

Rachele si accorge dei cambiamenti della sorella e di come cresca in fretta. Nel momento in cui Dafne rimpiange l'innocenza perduta, il mondo delle fate ormai lontano, e le comunica la sua volontà di abbandonare la scuola, Rachele riesce a trovare le parole adeguate per farle comprendere la necessità di affrontare la vita.

Rachele allora le raccontò, prima che si addormentasse, la fiaba della bella addormentata nel bosco e del principe azzurro che era venuto a risvegliarla con un bacio. Vedi, - le disse, per farla recedere dal suo proposito - se la principessa avesse continuato a dormire e a sognare, non avrebbe certo sofferto mai, ma non

Anche le Fate rappresentano un mito legato a quello della Dea Madre dal momento che il nome fata corrisponde al nome latino delle Parche (Fatae) che vuole indicare le divinità che controllano il fatum. 
avrebbe neppure avuto la gioia di vedere la bellezza delle cose reali, né conosciuto l'amore, con tutti i suoi rischi. (Madieri 1998: 273)

L'interpretazione della favola offerta da Rachele diventa, quindi, anche un invito all'emancipazione e all'indipendenza della donna, alla sua capacità e libertà di scegliere la propria strada, assumendosi la responsabilità delle proprie decisioni.

La sororità ritorna anche nella morte di Dafne, che strappata dal prato della sua radura, si ricongiunge alle sorelle in una ghirlanda di fiori che serve ad eleggere la Regina delle fate.

Sempre due sorelle, Maria e Sara, sono le protagoniste del romanzo Maria, dove ritroviamo, nuovamente, il legame sorella-mamma: «In casa erano rimaste lei e Sara, la figlia più piccola. Maria amava molto la sorella, dodici anni più giovane di lei, con un sentimento caldo e protettivo, quasi materno» (Madieri 2007: 16). Quando Maria abbandona la famiglia, Sara resta l'unico contatto e, nel momento in cui la sorella le telefonerà in cerca di aiuto e conforto, sarà pronta ad accoglierla a Trieste e ad offrirle, ancora una volta, la sua protezione.

Il tema della maternità in Maria è rappresentato come accoglienza e cura femminile e la protagonista si sdoppia nelle figure della mamma e della sorella. Gli uomini in questo romanzo sono soltanto delle ombre, alle volte terribili come quella del padre, oppure sfuggenti e senza nome, illusioni senza corpo, come nel caso degli amori di Maria. La genealogia femminile è invece molto forte: Maria accoglie la sorella come se fosse sua madre e cura suo nipote come se fosse suo figlio. In seguito andrà a vivere con la mamma in Puglia, l'unica persona per la quale è disposta a tornare alla sua terra natia. Questa posizione scambiabile e di identificazione si dimostra anche nella narrazione in terza persona e non in prima, per sottolineare il carattere collettivo e corale del romanzo, dove la storia della protagonista è fortemente legata alla storia delle altre donne, come già accadeva in Verde acqua e la Radura, dove l'autrice disegnava e completava il ritratto delle protagoniste attraverso gli altri personaggi femminili.

Il romanzo Maria va però più in là del circolo familiare, includendo in questa coralità anche la signora Savelli e Vittoria, alleate e mecenate di Maria e della sorella: «L'aveva presentata alla signora Savelli, a Vittoria e a Carlo ed erano tutte e due invitate una domenica a pranzo. Quel giorno Vittoria si era scatenata con i manicaretti $[\ldots]$ La signora Savelli aveva eccezionalmente sorriso un paio di volte» (Madieri 2007: 46).

\section{La lingua madre}

Un altro elemento che caratterizza Verde acqua è il riferimento alla 'lingua madre'. $\mathrm{Ne} L$ 'ordine simbolico della madre Luisa Muraro (1991), sottolinea l'importanza della lingua materna nella costruzione di una genealogia femminile, non soltanto strumento utile per decodificare ed interpretare il mondo, ma anche in grado di creare una mediazione, una condivisione, di costituire o rinsaldare i legami del cuore.

Marisa Madieri avverte il bisogno di recuperare la lingua materna, di ripartire proprio dal linguaggio, dalle parole apprese durante l'infanzia. In età adulta 
cercherà e ritroverà quella lingua da cui era stata in qualche modo esiliata, ristudiando il croato, appreso a Fiume da bambina e poi dimenticato. Così, la lingua si presenta come lo strumento per recuperare le proprie radici, per riappropriarsi della propria matria ${ }^{8}$.

Con Branko e Mike e tanti bambini slavi che frequentavano il mio giardino imparai rapidamente ad esprimermi in croato, ma poi altrettanto rapidamente dimenticai ogni cosa, dopo aver lasciato Fiume. Mi galleggiano nella memoria, come relitti in un oceano, solo alcuni frammenti di filastrocche infantili, di cui conosco solo il suono ma di cui mi sfugge il significato: cassezigonaiedè siraicrumpira zielahisciaseplema daziganche darozanche iossiselanema... Forse per inseguire questi significati perduti ho ripreso da due anni a studiare il serbo croato. (Madieri 1998: 32)

Anche Nonna Anka, che era diventata quasi mamma e moglie allo stesso tempo di Nonno Gigio, condensando due aspetti che anche nella figura della Dea Madre si fondono, recupera per lui la lingua madre.

Con lei Nonno Gigio regredì felicemente alla sua infanzia, ritrovando sua madre [...]. Nonna Anka infatti aveva per lui le cure più affettuose. Gli parlava in tedesco, lingua materna di mio padre, gli preparava piatti tipici della cucina serba ed ungherese, restituendogli i sapori della paprika, della cipolla, della cannella, dei semi di cumino e di papavero, ed infine lo faceva viaggiare instancabilmente. (Madieri 1998: 41)

Lo stesso farà zia Nina, la sorella di mamma Jole, che le sarà vicina, prendendosene cura negli ultimi momenti della sua malattia e, come per Nonna Anka e Nonno Madieri, userà la lingua materna, il dialetto, in questo caso, che le accomuna e le lega, per parlarle durante i loro incontri in ospedale.

Non si arrendeva al declino della sorella. Le parlava come sempre, le abbottonava la vestaglia lamentando ch'era qua e là macchiata di caffelatte, le portava delle paste, che le metteva in bocca a piccoli pezzetti. «Che bon», diceva la mamma, masticando lentamente con gli occhi chiusi. «Magna, Jole, magna che te fa ben, le ripeteva la zia, pulendole il viso con un fazzolettino immacolato». (Madieri 1998: 173)

\section{La rappresentazione della natura: il giardino e la terra-madre}

Il femminile matriarcale si ritrova nella rappresentazione della natura, attraverso le sue molteplici declinazioni. La metafora del giardino, che già in Verde Acqua giocava un ruolo importante, si ritrova nel realismo fatato de La Radura. Il giardino, in quanto luogo di nascita e di procreazione è presente costantemente nel

8 Il concetto di matria viene utilizzato da diverse scrittrici per poter indicare uno spazio di identità, condivisione, tradizione femminile che si possa contrapporre al concetto di patria. Si vedano ad esempio, Sendón (2006) e Marchetti (2014). 
mito della Grande Madre e nei suoi archetipi, a cominciare dal mito di Demetra e Persefone a quello delle Esperidi, ninfe che posseggono un prezioso giardino, dove custodiscono l'albero dalle mele d'oro.

Il giardino per Marisa Madieri risponde al mistero, al fantastico, alla scoperta di sé e del mondo. In Verde Acqua è il luogo dell'infanzia e delle prime esplorazioni, ma anche lo spazio dove ritrovare i ricordi e attingere al passato.

Il mio giardino che ho rivisto da adulta trovandolo misero e angusto, ai miei occhi infantili era il mondo intero, era l'avventura. Le sue siepi di ligustro era una foresta, i gatti che vi si nascondevano, i passeri, le formiche e le lucertole. Tutti gli animali dell'Eden, i sassi e i vetri colorati sparsi sul terreno tesori e pietre preziose che portavano alla abitazione della portinaia la scalinata di una reggia. (Madieri 1998: 30)

Questa particolare rappresentazione del giardino come cosmo in continua creazione, evoluzione e movimento, che ritornerà anche ne La Radura, sembra potersi ricollegare all'immagine della Grande Madre come demiurga, fonte di vita e protezione per tutti gli esseri viventi. Il giardino accompagnerà Marisa anche in età adulta e il suo fiorire sarà motivo di gioia e orgoglio, associandosi, inoltre, a momenti di vita, come nel caso del glicine, piantato dopo la nascita del figlio Paolo:

Tra qualche giorno il mio glicine fiorirà. L'ho piantato poco dopo la nascita di Paolo e ora è giunto a maturità. Fiorirà con ritardo, probabilmente perché si arrampica lungo la ringhiera del balcone esposto a nord. Ho contato otto piccoli racemi che di giorno in giorno si fanno più lunghi e più gonfi. Il giardino della mia casa di via Carpaccio è bello in questa stagione, grazie alle cure assidue e sapienti del Signor Zacchini. Il giovane mandorlo e la forsizia sono già sfioriti, ma la spirea e i tulipani sono ancora gloriosi. (Madieri 1998: 82)

Ne la Radura, la trasfigurazione di Marisa nella margherita Dafne le permetterà di osservare il suo percorso di crescita e cogliere la voce più intima e profonda della natura e di poterla filmare nel suo eterno divenire: «Era tutto uno sbocciare, crescere, strisciare, zampettare, svolazzare, scavare, brulicare, pareva di essere in un suk orientale» (Madieri 1998: 178).

Per Dafne, il giardino della radura rappresenta il vincolo con la terra madre e la morte significa la perdita di questo contatto.

Una cascata di capelli la sfiorò all'improvviso come una carezza e una piccola mano calda l'abbracciò alla base dello stelo. [...] Chiuse gli occhi e rabbrividì. Uno strappo un dolore intenso proprio al centro del creato. Si sentì sollevare, scuotere, stringere e infine adagiare, in un solido abbraccio con altre compagne su un cedevole giaciglio. Dov'era l'umida zolla materna? (Madieri 1998: 249)

Morire per Dafne significa anche aver compreso il senso ciclico della natura, il confine sottile che separa la vita dalla morte, che anche il mito della Grande Madre esemplifica. Dafne ha dato un senso alle parole di Rachele, che le aveva detto che i nonni riposavano distesi per terra e non si sarebbero rialzati e ha compreso anche le parole di Oscar, margherita giardiniere, che le aveva confessato, che prima o poi, 
sarebbe dovuto «tornare alla terra». Ora già sa rispondere a quell'interrogativo che la tormentava: «Come era possibile ritornare alla terra, se su di essa già stava ogni cosa? La terra era una madre generosa che ospitava nel suo grembo e reggeva sulla sua superficie tutte le creature, dalle più piccole, come le erbe e i fiori, alle più monumentali, come gli alberi. [...] Oscar doveva essersi sbagliato» (Madieri 1998: 189).

Una delle caratteristiche che contraddistingue la Grande Madre è quella di armonizzare elementi come la vita e la morte, ma anche il cielo e terra, la notte e il giorno, che la mitologia successiva andrà a contrapporre. La Grande Madre crea la natura, e contemporaneamente la racchiude in sé. Anche la maestra Venanzia aveva cercato di spiegarlo a Dafne, dicendole come «amore, morte, dolore, nascita, metamorfosi, tutto era legato in modo indissolubile» (Madieri 1998: 218), quella stessa immersione/fusione con la natura che Dafne aveva vissuto nel sogno che racconta all'amica Celeste.

Sai Celeste ieri ho fatto un sogno bellissimo e strano. Ero su un grande prato ceruleo, come su un cielo rovesciato, in cui al posto delle margherite crescevano tante stelle. Anche io ero una stella e anche tu. Eravamo adagiate su un fondo molle, nel quale era facile sparire, sprofondando come in un quieto sonno. Ma io non volevo spegnermi e cercavo di rimanere in superficie. D'improvviso si è alzato un forte vento che, vincendo ogni mia resistenza, mi ha sollevata in un vortice. Mi portava in alto lentamente, in larghi cerchi, oltre le nubi, verso oscure profondità, dove si spalancava come un pozzo lucente, il volto bianco della luna. E io giravo giravo precipitando in quell'abisso, fino allo stordimento, fino a non sapere più se ero nuvola, vento, margherita o stella. (Madieri 1998: 207)

Non riuscire a creare la propria discendenza e genealogia, per continuare ad essere legata alla radura, è forse l'unico rammarico che resta alla margherita Dafne quando viene strappata dal suo prato. Sarà Celeste, l'amica del cuore, a farlo per lei, in un simbolico passaggio di testimone, che salderà per sempre il legame tra le due amiche: «Era questa la fine del tempo? E i frutti e i semi? Oh Celeste falli anche per me. Sì - le parve di udire - anche per te, amica mia, anche per te» (Madieri 1998: 250).

Il riferimento al giardino, come terra di appartenenza, si avverte, infine, anche in un 'romanzo acquatico' come Maria, e quando la protagonista decide di tornare a vivere dalla madre in Puglia, il loro ricongiungimento avviene proprio nell'orto di casa.

Quando, nel tardo pomeriggio, arrivò vicino a casa, con le due valigie e la borsa a tracolla, vide nell'orto una figura scura, china a terra. Chiamò con voce tremante dalla commozione. La madre si levò a guardare, stringendo un poco gli occhi miopi, e apparve piccola, fragile in quello spazio così grande e sotto quel cielo così perentoriamente azzurro. Maria le corse incontro e l'abbracciò. (Madieri 2007: 55) 


\section{La rilettura del mito: Dafne, la Sirena e altri archetipi della dea madre}

Un'altra costante nella narrativa di Marisa Madieri è il riferimento al mito che si sviluppa, ora attraverso il riferimento a miti concreti come quello di Dafne nella $L a$ Radura e delle Sirene nel racconto Riccardo e la Sirena, o per mezzo di archetipi della Grande Madre come nel caso di Mauroa in La Conchiglia.

Nel caso de La Radura la rilettura/riscrittura di un mito classico come quello di Dafne rappresenta un nuovo aspetto del matriarcale femminile. La libertà e l'indipendenza della Ninfa, che seppe respingere Apollo e si trasformò in alloro per sfuggirgli, è la stessa che caratterizza Dafne-margherita che, però, corre verso un amore ancora da scoprire. Ma se Dafne-ninfa, una volta trasformata in albero di alloro, resterà ancorata ad uno stato di passività, bellezza da contemplare e le sue foglie serviranno ad Apollo per cingersi il capo durante altre rincorse e violenze, Dafne-margherita diverrà con le compagne corona della regina delle fate, in un contrappasso forse tragico e crudele, ma dopo aver completato il suo percorso, dopo aver attraversato il suo mondo delle fate ed essere entrata nel mondo reale, fatto di gioie, ma anche sofferenze.

Un'altra rappresentazione della Grande Madre è quella della vecchia Mauroa che vive nella grotta di Kalenga, nel racconto La Conchiglia. Mauroa racchiude in sé le caratteristiche della maga e della strega. Si tratta, dunque, di un personaggio che incute timore, perché costituisce una rottura con la normalità e ciò la costringe a vivere ai margini, esiliata dalla comunità e dalla cosiddetta civiltà.

Mi incuteva reverenza e timore quella creatura rugosa, ossuta, con i seni svuotati, gli occhi mobili e penetranti e i lunghi capelli grigi che le scendevano a mezza schiena radi e disordinati. [...] Era gentile d'animo ancorché indurita nei modi per gli affanni. Amava i bambini che non si lasciavano ingannare dal suo aspetto e la avvicinavano senza timore, anche dopo il suo volontario esilio dalla comunità. Era sempre pronta a vezzeggiarli e a dividere con loro le povere cose che possedeva. (Madieri 1998: 261)

Con il tempo, Mauroa saprà conquistare il rispetto e il riconoscimento di saggia, da parte della comunità, ma sceglierà comunque di continuare a vivere nella sua grotta, perché custodisce la sua essenza e rappresenta il luogo in cui si sente maggiormente protetta.

Mauroa aveva un talento straordinario per i tatuaggi del corpo e la pittura della faccia, talento che si era rivelato durante le frequenti gare che si tengono tra clan. Molti cominciarono a farsi dipingere da lei il viso per le cerimonie, ricompensandola con doni se il risultato li soddisfaceva. Si conquistò così negli ultimi anni della sua vita, una modesta agiatezza e il ruolo riconosciuto di persona saggia, rispettata da tutti. Ma nessuno riuscì mai a convincerla ad abbandonare la grotta per ritornare nel villaggio. (Madieri 1998: 261)

Anche in Riccardo e la Sirena, troviamo una reinterpretazione di un mito classico. Riccardo è un bambino che, dopo la morte della madre, va ad abitare nella casa della nonna, con il padre che, abbandonato il lavoro di elettricista, si trasforma in pescatore. Una notte, mentre lo accompagna a pesca, Riccardo sente per la prima 
volta parlare delle sirene e, da quel momento, decide di volerne incontrare una. È così che, durante le sue immersioni subacquee, conosce Aia e parla con lei:

Qual è il vostro nome, signora sirena? [...]

Aia mi chiamo, figlia del mare, e sono qui che ti aspetto da molto tempo.

[...] Sei un bravo bambino, Riccardo, sono fiera di te. [...] Nulla sfugge a noi abitatori delle profondità. Le storie degli uomini sono tutte scritte sul fondo del mare. (Madieri 1998: 285)

Gli incontri si susseguono e accanto ad Aia, Riccardo sembra ritrovare la felicità e la serenità che sembravano perdute. Chiacchera, nuota e gioca con lei fino a quando, un giorno, la sirena gli annuncia la sua partenza, non prima di un ultimo dono: «Devo andarmene, gli disse, ma ti voglio lasciare un ricordo. Gli porse una graziosa cornice ornata di gusci di chiocciole e piccole conchiglie dall'interno madreperlaceo. Ogni volta che la guarderai, io ti sarò vicina» (Madieri 1998, 285).

Dopo la partenza di Aia, Riccardo si ammala, la febbre alta sembra non volere scendere e durante le notti la invoca invano, vorrebbe tornare a nuotare con lei, fino a quando il papà e la nonna scoprono sul tavolino vicino al letto la cornice di conchiglie. Quella cornice che papà Matteo credeva di aver smarrito e che era un ricordo lieto di un viaggio con la moglie Marina (anche nella scelta del nome ritorna l'elemento acquatico), contiene una vecchia fotografia di Riccardo con la mamma.

Nella fotografia Riccardo aveva un cappellino bianco in testa e giocava sulla spiaggia con paletta e secchiello. Vicino a lui la mamma era distesa su un fianco appoggiata ad un gomito. I lunghi capelli neri apparivano un po'scompigliati dal vento, il profilo del suo corpo rivelava morbide rotondità che si assottigliavano nel rilievo delle gambe allungate, tenute una leggermente sull'altra, sicché alla fine erano visibili una sola caviglia e un solo piede. Pareva una bellissima sirena. (Madieri 1998, 286)

Nell'Antichità classica, le sirene erano associate agli inferi e alla morte, e in alcuni casi venivano considerate le incaricate di condurre i morti all'Ade. Anche l'iconografia che le riguardava metteva in risalto la loro connotazione mostruosa, e le raffigurava come le Arpie, con volto di donna e corpo di rapace. Il mito che ne racconta la nascita le vuole figlie di Acheloo, divinità fluviale e nate dal suo sangue, quando questi, in un combattimento, venne ferito da Eracle. Dal dodicesimo canto dell'Odissea diventeranno, inoltre, un emblema della seduzione maligna, del femminile malvagio e pericoloso, dal momento che con il loro canto cercano di attrarre i marinai, per condurli, in realtà, alla morte ${ }^{9}$.

Marisa Madieri ribalta il mito e conferisce ad Aia sembianze materne, trasformando il femminile mortifero in femminile che accoglie e protegge. La ricerca della Sirena da parte di Riccardo rappresenta un passaggio determinante nel suo processo di crescita, come per Dafne lo era la ricerca delle fate. Entrambi, sebbene in maniera diversa, troveranno una risposta ai loro quesiti, Dafne

9 Una ricostruzione dell'evoluzione del mito della sirena dalla Grecia antica fino alla contemporaneità si trova in Bettini e Spina (2007). 
attraverso la bambina, che con una corona di margherite sarà eletta Regina delle fate, e Riccardo attraverso l'incontro simbolico-fantastico con la madre.

L'immersione nel fondale del mare, alla ricerca della sirena Aia, per Riccardo diventa quasi un fluttuare nel liquido amniotico, come quello de Il bambino con le ali, e gli permette di ritrovare il femminile matriarcale perduto.

\section{Acqua, mare, grotte e conchiglie}

Il continuo richiamarsi all'acqua e al mare ${ }^{10}$ contraddistingue tutta la produzione di Marisa Madieri e rappresenta l'ennesima manifestazione del femminile matriarcale. Quello con il mare, in particolar modo, era un vincolo profondo e vitale per la scrittrice, come ricorda anche Claudio Magris:

Nelle pagine di Marisa Madieri c'è molta acqua, molto mare. Paesaggio anche di lontananza e di solitudine, di malinconia e di tragedia, ma soprattutto di pienezza e di abbandono, di esistenza condivisa e vissuta a fondo, come un'estate gloriosa ed inesauribile. [...]. Marisa amava moltissimo il mare, ne sentiva fortemente il legame e il rapporto con l'amore; abbandonarsi alle onde, sentire il loro rumore, o lasciarsi portare dal loro scorrere, guardare anche per ore il rincorrersi e l'infrangersi delle creste bianche era per lei una delle esperienze della felicità, quella più simile alla pienezza amorosa e alla grazia dell'infanzia-infanzia dell'individuo e della umanità intera, che provengono dall'acqua, anche se spesso lo dimenticano. Il paesaggio marino è lo scenario dell'identificazione amorosa, del ritrovamento di sé, di quell'eros che sottaciuto ed alluso, attraversa le sue pagine, di una vita vissuta come un'estate gloriosa. (Magris 1998: 303)

L'acqua nella rappresentazione mitica è soprattutto madre, perché è origine della vita, perché è dall'acqua che nascono tutti gli esseri, e anche nella sua etimologia si avverte questa eredità, come nel caso del francese dove due parole come mare (mèr) e madre (mère) si pronunciano allo stesso modo. L'acqua racchiude in sé gli stessi dualismi presenti nel mito della Grande Madre, dal momento che, a seconda delle cultura e dei miti, è considerata elemento vitale, ma anche letale, origine e fine, risoluzione e dissoluzione e contemporaneamente mediazione dei contrari (Ortiz-Osés 1993).

Nel romanzo Maria, il rumore dell'acqua, e il desiderio di toccare il mare, accompagnano la protagonista durante le sue passeggiate e il suo vissuto diventa liquida temporalità. L'acqua scandisce tutte le sue vicende e anche i suoi pensieri, $\mathrm{i}$ ricordi e le metafore rimandano e sono riportati all'acqua ed ad un contesto ed un linguaggio marino: «solo un ricordo si fece strada nel suo animo e spezzò in mille risonanze il buio lavico in cui era immersa, come una prua che frantuma in schiume, gocce e cascate l'inerte superficie del mare» (Madieri 2007: 33).

Anche il sentimento di protezione di Maria nei confronti della sorella, che poi si trasferirà sul figlio di Sara, che su sua richiesta chiamerà Marco (il nome di quel figlio che avrebbe voluto ma al quale ha dovuto rinunciare?) è legato all'acqua. Marco si presenta come un puer, cioè un figlio legato alle due figure femminili

10 Marisa Madieri dedicò al tema anche un saggio (Madieri 1989: 64-71). 
della mamma e della zia, senza avere una figura paterna di riferimento. La sua ontogenia non è soltanto biologica, ma spirituale. Infatti, le sue favole favorite e di riferimento appartengono inequivocabilmente al regno del femminile acquatico: «favole che avevano come protagoniste conchiglie, ricci, stelle marine, pesci grandi ma buoni o pesci piccoli dai colori vivaci. Tutti abitavano in misteriose grotte azzurre o tra stupendi coralli vermigli» (Madieri 1998: 52).

Altri simboli matriarcali che ricorrono sia ne La Conchiglia sia in Maria sono le grotte, le caverne e le gallerie. La loro forma concava e profonda è un'espressione della maternità perché richiama l'utero della madre. In vari miti ed episodi dell'Odissea questi elementi sono associati al femminile. Si pensi ad esempio a luoghi del testo omerico come l'antro «amabile e oscuro» delle Naiadi, la grotta di Circe, la baia delle Sirene ${ }^{11}$. In Maria, ritorna il riferimento al simbolo della grottacaverna, quando l'autrice racconta le passeggiate lungo il mare della protagonista.

Più avanti, lungo un ampio tratto di costa, fino al bivio che conduceva da una parte alla strada costiera e dall'altra al castello di Miramare, ciclopici massi erano posti come frangiflutti a ridosso della riva. Il mare iniziava oltre questo sbarramento, ma s'infiltrava tra roccia e roccia creando piccoli bacini neri, conche d'acqua bassa e trasparente, ristagni olivastri. Il rumore dell'acqua di queste caverne artificiali era vario, orchestrato sui diversi livelli delle maree, sull'impeto delle onde e della risacca. (Madieri 2007: 6)

All'immagine della grotta è strettamente collegata anche quella della conchiglia. Come la caverna, anche la conchiglia rappresenta un altro elemento che fa parte della simbologia matriarcale, e nella sua forma richiama l'organo sessuale femminile ${ }^{12}$.

Da una conchiglia nasce la Venere di Botticelli, di cui l'autrice, come ricorda in Verde Acqua, conserva una riproduzione appoggiata ai libri della sua camera da letto: «mi piace incontrare con lo sguardo i serti di fiori che ornano l'abito morbido e leggero della Primavera e osservare la fossetta sul mento di Venere che, in una luce fredda e mattutina, sorge bianca sulla conchiglia tra fremiti d'onde e aliti di vento» (Madieri 1998: 158). Una conchiglia è il regalo che il protagonista del racconto omonimo dona a Naipuni, sua futura sposa, e «con anelli di conchiglie tintillanti» ai polsi e alle caviglie, sempre Naipuni balla durante una festa. Una cornice ornata di gusci di chiocciole e piccole conchiglie è il dono che Riccardo riceve dalla sirena Aia, in Riccardo e la sirena, e, infine, una conchiglia bianca e luminosa è quella che a Maria sembra di vedere in una piccola grotta e che sogna di poter raggiungere un giorno.

In una di queste profonde fessure le pareva di intravedere qualcosa di luminoso e cangiante, un inatteso arcobaleno sotterraneo. Forse era una conchiglia che una tempesta aveva strappato ai suoi fondali, o forse si trattava semplicemente di un

11 Sul significato della simbologia della grotta si veda, ad esempio, Marchetti (2011).

12 Il potere apotropaico della conchiglia è evidenziato dal fatto che una particolare conchiglia, la cosiddetta conchiglia cauri, bianca e dalla caratteristica forma di vulva, si affermò e fu utilizzata a partire dal Neolitico come moneta. Ancora oggi presso alcune popolazioni africane la cauri si usa per offrire donazioni durante cerimonie funebri o occasioni rituali. 
varco più ampio verso il chiarore del mare tra il groviglio scomposto dei massi. Maria preferiva pensare alla conchiglia che restituiva la magia delle luci catturate nelle profondità marine. Le pareva perfino di scorgerne il guscio bianco e spiralato. Un giorno sarebbe scesa tra quelle rocce e avrebbe cercato di prenderla in mano per osservarla da vicino. (Madieri 2007: 70)

Quella stessa conchiglia che, una volta ritornata a Trieste dalla Puglia, prova a cercare nuovamente, ma non riesce a ritrovare. Nel caso di Maria, la simbologia della grotta e della conchiglia diventano ancora più significative, dal momento che attraverso la ricerca di questo contatto con elementi legati alla fertilità sembra rincorrere una maternità perduta. Non poter ritrovare la conchiglia la mette di fronte, definitivamente, all'impossibilità di essere madre. Solo il contatto con il mare, ancora una volta, sembra poterle offrire conforto: «D'improvviso sentì il desiderio di toccare il mare [...]. Immerse la mano nell'acqua, in un punto in cui era più chiara, si bagnò la fronte e gli occhi, lentamente, un gesto battesimale [...] le piaceva sentire sulle palpebre le gocce salate, come lacrime necessarie» (Madieri 2007: 68).

Quello di Maria appare come un rituale di purificazione alla ricerca della fertilità e insieme un rito di sacrificio, un abbandonarsi, un fondersi con il maremadre, in un abbraccio eterno, in cui dolore e gioia si mescolano e dove può ritrovare riflessi i volti amati e conosciuti e anche il volto di quel bambino rincorso a lungo, e che fino ad allora aveva solo potuto immaginare.

La riva era vuota e il mare s'era fatto immobile e scuro, un grembo da cui proveniva ogni vita e in cui esistevano forse tutte le generazioni, passare e future. [...]. L'acqua era l'abisso del tempo, il grumo doloroso del vivere. Ma i fondali erano ancora soffusi di un debole chiarore [...]. Quel gioco di riflessi disegnava a tratti i lineamenti di Sara, della madre, di Marco, cancellandoli subito in un fluido divenire. Ma non era uno svanire doloroso. Le loro immagini si trasformavano, non si perdevano. Diventavano esse stesse acqua, onde, dolce oscurità. [...] Infine riconobbe - sfuggente eppur vicino - il volto disperatamente amato, sempre cercato, mille volte immaginato. Delicato, di una bellezza essenziale e dolente. E le sorrideva. Tutto era così facile ora. (Madieri 2007: 6970)

Come Maria, che si riflette tra i volti che scorge tra le onde, allo stesso modo, Marisa Madieri nei suoi racconti, intreccia ed innesta nuove relazioni, costruisce nuovi legami e ne recupera di antichi. La sua opera si snoda attraverso un variegato mosaico di figure femminili e leggendo le sue pagine è possibile seguire le tracce di simboli e riferimenti matrilineari. Il viaggio verso le altre donne, si trasforma, allora, in un viaggio, anche, verso se stessa e la memoria può diventare «il luogo del tributo di gratitudine e di amore verso chi, senza eroismi e gesti eccezionali, ma con la semplicità della vita, ci ha amato e aiutato a vivere» (Madieri 2015: 172). 


\section{Riferimenti bibliografici}

Anedda, Antonella (1998): «Il gioco della vita in un grano di sabbia», Il Manifesto, 22 novembre.

Bachofen, Johan Jacob (1861): Das Mutterrecht. Eine Untersuchung über die Gynaikokratie der alten Welt nach ihrer religiösen und rechtlichen Natur, 2 voll., Basel, Schwabe. [Tr. it. di G. Schiavoni, Il matriarcato. Ricerca sulla ginecocrazia nel mondo antico nei suoi aspetti religiosi e giuridici, 2 voll., Torino, Einaudi, 1988].

Benelli, Graziano (1993): «Conversazione sulla Radura», Lingua e letteratura, 20, pp. 173176.

Benussi, Cristina; Semacchi Gliubich, Graziella (2011): Marisa Madieri. La vita, l'impegno, le opere, Firenze, Ibiskos editrice.

Bettini, Maurizio; Spina, Luigi (2007): Il mito delle sirene: immagini e racconti dalla Grecia a oggi, Torino, Einaudi.

Bianchi, Graziano (2003): La narrativa di Marisa Madieri, Firenze, Le Lettere.

Branca, Vittore (1992): «La fiaba di Dafne», Messaggero Veneto, 27 ottobre.

Branca, Vittore (1996): «La mamma-sirena di tutte le anime orfanelle», Il Sole-24 Ore, 15 settembre.

Carminati, Maria (2006): «Marisa Madieri e la letteratura femminile dell'esodo», La Battana, 160 (aprile-giugno), pp. 175-191.

Carminati, Maria (2007): «Postfazione», in Marisa Madieri, Maria, Milano, Archinto, pp. 71-91.

García Gual, Carlos (2014): Sirenas. Seducciones y metamorfosis, Madrid, Turner Noema.

Gerbaz Giuliano, Corinna (2011): «La produzione letteraria di Marisa Madieri», Quaderni d'italianistica, XXXII (1), pp. 65-81.

Gerbaz Giuliano, Corinna; Mazzieri Sanković, Gianna (2013): Non parto, non resto...: $i$ percorsi narrativi di Osvaldo Ramous e Marisa Madieri, Trieste, Deputazione di storia patria per la Venezia Giulia.

Gimbutas, Marija (1989): The language of the Goddess, London, Thames and Hudson. [Tr. it. Di Nicola Crocetti, Il linguaggio della Dea. Mito e culto della Dea Madre nell'Europa neolitica, Milano, Longanesi, 1990].

Guagnini, Elvio (1994): «La radura di Marisa Madieri», in Frances De Nicola, Pier Antonio Zannoni (a c. di), Scrittrici d'Italia. Atti del Convegno Nazionale di Studi Città di Rapallo, 14 maggio 1994, Genova, Costa \& Nolan, pp. 114-115.

Guagnini, Elvio. (2004): «Marisa Madieri la musica dolce dei ricordi», Il Piccolo, 7 gennaio.

Guagnini, Elvio (2014): «Un giardino per Marisa Madieri», Archeografo Triestino, Serie IV, LXXIV, pp. 181-184.

Guagnini, Elvio; Ivancic, Bruna; Ladrón de Guevara, Pedro Luis; Storti, Anna (eds.) (2014): Quaderno della mostra 'Piccole gocce nell'oceano del vissuto': Marisa Madieri e l'emozione della scrittura (Trieste, 10-10 Dicembre 2014), Trieste, Archivio e Centro di Documentazione della Cultura Regionale.

Ioli, Giovanna (1992): «La favola di Dafne piccola margherita», Il Nostro Tempo, 47, 20 dicembre.

Irigaray, Luce (1989): Sessi e genealogie, Milano, La Tartaruga.

Ladrón de Guevara, Pedro Luis (2012): «Traducción y representación de una feminidad que no siempre existió: el mito de las sirenas», in Milagros Martín Clavijo, Salvatore 
Bartolotta, Michela Caiazzo, Daniele Cerrato (a c. di), La voces de las diosas, Sevilla, Arcibel Editores, pp. 737-765.

Ladron De Guevara, Pedro Luis (2014): «Identità ed esodo nell'opera di Marisa Madieri», Rivista di Letteratura Italiana. L'esodo giuliano dalmata nella letteratura, Pisa Roma, Fabrizio Serra editore, pp. 61-66.

Ladrón de Guevara, Pedro Luis; Hernández Gonzalez, María Belén; Zografidou, Zosi (eds.) (2015): Marisa Madieri: Escritoras del éxodo y del exilio, Murcia, Editum.

Madieri, Marisa (1989): «Acqua è poesia - Water is poetry», Ciga Hotels Magazine, XVII (81), pp. 64-71.

Madieri, Marisa (1998): Verde Acqua, La Radura e altri racconti, introduzione di Ermanno Paccagnini, postfazione di Claudio Magris, Torino, Einaudi.

Madieri, Marisa (2000): Verde agua, trad. di Luisa Bergalli, postfazione di Claudio Magris, Barcelona, Minúscula.

Madieri, Marisa (2007): Maria, ed. Di Maria Carminati, Milano, Archinto.

Madieri, Marisa (2015): «Documentos inéditos de Marida Madieri», in Pedro Luis Ladrón de Guevara, María Belén Hernández González, Zosi Zografidou (a c. di), Marisa Madieri: Escritoras del éxodo y del exilio, Murcia, Editum, pp. 163-185.

Magris, Claudio (1998): «Postfazione», in Marisa Madieri, Verde Acqua, La Radura e altri racconti, Torino, Einaudi, pp. 288-304.

Magris, Claudio (1998): «Postfazione», in Marisa Madieri, La Conchiglia e altri racconti, Milano, Scheiwiller, pp. 62-73.

Marchetti, Laura (2011): La luce della notte. Modelli per una didattica delle culture, Bari, Palomar.

Marchetti, Laura (2014): La Fiaba, la Natura, la Matria. Genova, Il Melangolo.

Monmany, Mercedes (2001): «Una historia del tiempo», Revista de libros, 53, pp. 42-43.

Muraro, Luisa (1991): L'ordine simbolico della madre, Roma, Editori Riuniti.

Neumann, Eric (1956): Die Grosse Mutter. Ein Archetyp des Grosses Weiblichen, Zürich, Rhein-Verlag. [Trad it. di Antonio Vitolo, La grande madre. Fenomenologia delle configurazioni femminili dell'inconscio, Roma, Astrolabio, 1981].

Ortiz-Osés, Andrés (1993): Las claves simbólicas de nuestra cultura. Matriarcalismo, patriarcalismo, fratriarcalismo, Barcelona, Anthropos.

Paccagnini, Ermanno (1998): «Introduzione», in Marisa Madieri, Verde Acqua, La Radura e altri racconti, Torino, Einaudi, pp. 3-17.

Paccagnini, Ermanno (2014): «Il valore della memoria e il diritto all'emozione», in Elvio Guagnini, Bruna Ivancic, Pedro Luis Ladrón de Guevara, Anna Storti (a c. di), Quaderno della mostra Piccole gocce nell'oceano del vissuto. Marisa Madieri e l'emozione della scrittura (Trieste, 10-10 Dicembre 2014), Trieste, Archivio e Centro di Documentazione della Cutura Regionale, pp. 7-10.

Pampaloni, Geno (1987): «La scrittura 'acqua e sapone'», Il Giornale, 7 giugno.

Pellegrini, Ernestina (1995): «Le radici della nostra debolezza. "La radura" di Marisa Madieri», in Ernestina Pellegrini, Le città interiori. In scrittori triestini di ieri e di oggi, Bergamo, Moretti e Vitali, pp. 131-154.

Pellegrini, Ernestina (2000): «Per Marisa Madieri. I racconti rasoterra», Antologia Vieusseux, 16-17, pp. 173-184.

Pellegrini, Ernestina (2001): «La trasparencia de Marisa Madieri. Autobiografía como despedida», in Mercedes Arriaga Flórez, Dolores Ramirez Almazán (a c. di), 
Representar/Representarse. Firmado Mujer. Homenaje a Zenobia Camprubi, Huelva, Diputación de Huelva, pp. 605-618.

Ramoneda, Josep (2003): «Tiempo Condensado», El País, 4 novembre 2000; rist. in Ramoneda, Josep, Del tiempo condensado. Globalización, Immaginación, Terrorismo, Cultura, Barcelona, DeBolsillo, pp. 422-423.

Russo, Fabio (2007): «Marisa Madieri: una riflessione», Lettere italiane, 59 (1), pp. 111123.

Sendón de León, Victoria (2006): Matria: El horizonte de lo posible, Madrid, Siglo XXI.

Sgorlon, Carlo (1987): «Quella scelta di rimanere italiani», Il Piccolo, 19 settembre.

Siciliano, Enzo (1999): «Le radici strappate di Marisa Madieri», La Repubblica, 30 marzo.

Trader, Uta (2000): «Voci di donne nell'ultima Marisa Madieri», Antologia Vieusseux, 1617, pp. 192-196.

Venucci Maržo, Patrizia (2010): «Omaggio a Marisa Madieri. Intervista a Claudio Magris», La Battana, 176, aprile-maggio.

Zografidou, Zosi (2014): «Bibliografia de Marisa Madieri», in Pedro Luis Ladrón de Guevara, María Belén Hernández González, Zosi Zografidou (a c. di), Marisa Madieri: Escritoras del éxodo y del exilio, Murcia, Editum, pp. 195-203. 